\title{
Practical Application of Pediatric Hospital Medicine Workforce Data. In Reference to: "Pediatric Hospitalist Workload and Sustainability in University-Based Programs: Results from a National Interview-Based Survey"
}

\author{
Lindsey C Douglas, MD, MSCR ${ }^{1,2 *}$, Karen M Wilson, MD, $\mathrm{MPH}^{1,2}$
}

'Department of Pediatrics, Icahn School of Medicine at Mount Sinai, New York, New York; ${ }^{2}$ Kravis Children's Hospital, New York, New York.

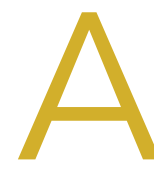

s leaders of a new Pediatric Hospital Medicine program in New York City, we were pleased to read the Brief Report from Dr. Fromme and colleagues, "Pediatric Hospitalist Workload and Sustainability in University-Based Programs: Results from a National Interview-Based Survey."

Although the study has greatly assisted us in developing our program, the manuscript lacked some data necessary for workforce planning. The authors report census caps for a majority of programs, but neither the actual number of patients in each cap nor whether programs with caps reported an association with patient safety or program sustainability. In addition, although overnight pager calls were calculated in median hours,

*Corresponding Author: Lindsey C. Douglas, MD; E-mail: lindsey.douglas@ mssm.edu; Telephone: 212-241-1251.

Received: November 2, 2018; Accepted: December 22, 2018

๑) 2019 Society of Hospital Medicine DOI 10.12788/jhm.3149 there were no data on whether nights were weighted or alternate staffing models were used for overnight pager calls.

While the article will help guide our field's continued understanding of our workforce, without additional detailed data, we found that we were unable to apply staffing models practically in the real world to our new program. Pediatric Hospital Medicine is one of the fastest growing fields in medicine; however, support of new programs and sustainability of existing ones, require benchmark details to create proposals that are acceptable to both hospital and university administrators while maintaining workforce sustainability.

\section{Disclosures: Drs. Douglas and Wilson have nothing to disclose.}

\section{Reference}

1. Fromme HB, Chen C, Fine B, Gosdin C, Shaughnessy E. Pediatric Hospitalist Workload and Sustainability in University-Based Programs: Results from a National Interview-Based Survey. J Hosp Med. 2018. 13:702-705. 